\title{
Identification of the Most Commonly Used Laboratory Techniques in Regenerative Medicine: A Roadmap for Developing a Competency Based Education Curriculum
}

\author{
Stephen L. Rego, ${ }^{1}$ Cheryl Burrell, ${ }^{1}$ Melissa Nielsen, ${ }^{1}$ \\ Tatjana Grove, ${ }^{1}$ Amritha Kidiyoor, ${ }^{2}$ Vatashea Flournoy, ${ }^{1}$ Cheri Silverman, ${ }^{1}$ \\ Shawn Hill, ${ }^{1}$ Grady Beard, ${ }^{1}$ Dwaine Davis, ${ }^{1}$ Mehrdad Tajkarimi, ${ }^{1}$ \\ Courtney Harrington, ${ }^{1}$ Janice Byrum, ${ }^{1}$ and Michael Ayers ${ }^{1}$ \\ ${ }^{1}$ Math, Science and Technology Department, Forsyth Technical Community College, Winston-Salem, NC, USA \\ ${ }^{2}$ Wake Forest Institute for Regenerative Medicine, Wake Forest School of Medicine, Winston-Salem, NC, USA \\ Correspondence should be addressed to Stephen L. Rego; srego@wakehealth.edu
}

Received 10 April 2016; Revised 30 August 2016; Accepted 22 September 2016

Academic Editor: Shu-Sheng Liaw

Copyright (C) 2016 Stephen L. Rego et al. This is an open access article distributed under the Creative Commons Attribution License, which permits unrestricted use, distribution, and reproduction in any medium, provided the original work is properly cited.

\begin{abstract}
Here, we are proposing and testing the use of literature reviews as a method to identify essential competencies for specific fields. This has implications in how educators develop and structure both traditional and competency based curricula. Our focus will be on utilizing this method to identify the most relevant and commonly used techniques in the field of regenerative medicine. This publication review method may be used to develop competency based education (CBE) programs that focus on commonly utilized skills. CBE is an emerging trend in higher education that will greatly enhance student learning experiences. CBE works by providing students with field specific skills and knowledge; thus, it is imperative for educators to identify the most essential competencies in a given field. Therefore, we reason that a literature review of the techniques performed in studies published in prevalent peer reviewed journals for a given field offers an ideal method to identify and rank competencies that should be delivered to students by a respective curriculum. Here, we reviewed recent articles published on topics in the field of regenerative medicine as a proof of concept for the use of literature reviews as a guide for the development of a regenerative medicine CBE curriculum.
\end{abstract}

\section{Introduction}

1.1. Competency Based versus Traditional Education. It has long been perceived that CBE programs were an excellent approach for enhancing student's education experience and future success $[1,2]$. CBE is defined as a structured approach to providing students with the necessary knowledge and skills needed to perform a task to a certain standard $[1,3]$. Although many educational institutions and programs support the utility of CBE strategies, a significant lag in deploying this type of education has been observed $[4,5]$.

The practical benefits of traditional cohort based education from a university's standpoint are apparent since educators deal with groups of students moving at the same pace rather than individual students moving at their own pace [6].
Traditional education can be easily fit into a semester based and credit hour system. In a traditional setting instructors know exactly when material must be available to students and can prepare lectures accordingly. It may even be argued that a traditional grading system is easier to implement than in the setting of CBE. Although this may be true under current models used to provide education, we anticipate that the "inconveniences" associated with CBE can be addressed by modifying our practices.

If appropriate methods are developed to deliver CBE programs, there is potential to increase the overall efficiency of education. First, a CBE education system allows an institution to take into account a student's previous learning experience if it proves beneficial to their understanding and performance of a specific competency [6-10]. This would decrease the time 
and cost of education which, in a traditional setting, does not depend on the experiences students bring with them to a program. Furthermore, since CBE will allow students to obtain a competency at their own pace this could decrease the amount of time students require to complete a program.

By definition a CBE course or program must have clearly defined, concrete, and measurable objectives $[1,5,7-9,11]$. This means assessment methods should be able to reflect whether or not students have obtained a competency and can move on to the next course. Also inherent to CBE programs is an "individualized" learning experience. Methods that have been identified to provide individualized learning include multiple modes of learning activities and an online format that allows students to complete courses at their own pace with added flexibility compared to traditional settings [1].

1.2. Methods to Identify Competencies. With the methods for development and delivery of general CBE programs discussed in length in the literature and elsewhere $[2,3,5,6,9$, 11] a void remains in how to identify the specific competencies or techniques students require in order to become proficient in a specific field. One option is to start with what is already available in the traditional course outlines, text books, and laboratory manuals [12-15]. Using this method one could first identify the traditional courses a student should take to obtain a degree in a specific field, for example, regenerative medicine, and extract the competencies covered in the course outlines $[13,16]$. To fit within the framework of CBE, the text books and lab manuals associated with the traditional courses may or may not be utilized but materials that reflect their content would be developed in a manner that fits the CBE model (online, multiple modes, etc.). This approach, although convenient, may not be in the best interest of the students, potential employers or the institutions since the most recent competencies associated with a certain field may not be covered in the currently available courses $[17,18]$. Furthermore, additional content that may not be relevant to a given field may be included in these materials making this top down approach less than ideal.

Another method is to survey experts in the specific field in which you wish to train students [7-9]. Identifying competencies in this way is beneficial because the competencies you identify are coming directly from the environment in which your graduates will likely work and potentially the individuals your graduates will work with. If, for example, an institution wished to develop a regenerative medicine curriculum, they could survey researchers at the Wake Forest Institute of Regeneration Medicine or the Center for Regenerative Medicine at the Mayo Clinic. A disadvantage to this method would be its tediousness, reliance on potentially unwilling participants, and potential response bias [9, 19-21].

The approach implemented here was the identification of the most common and widely used techniques through a literature review of articles published in highly regarded peer reviewed journals. This approach is less tedious and has the potential to be more objective and less biased than other approaches, including surveying experts in the field. Furthermore, this literature review will not be confined by location or willingness of researchers to respond. The primary criteria for identifying articles used in this review will be the relevance of the articles to the field of regenerative medicine and the quality of journal where articles were published. Recent relevant articles were identified by performing a PubMed search for articles that contained the terms "regenerative medicine", "stem cells", "biomaterials", and "tissue engineering" and identifying original research articles that were published in the last year. The journals were screened and utilized in this review only if they had an impact factor or were highly cited but not around long enough to have a designated impact factor. This will allow the identification of the most important and influential competencies utilized in the field and since these articles have been peer reviewed we can be more confident that these competencies are accepted by the field. The focus of this paper is to identify a list of the most relevant and commonly used competencies practiced in the field of regenerative medicine which could be used as a guide to develop a competency based curriculum for regenerative medicine.

\subsection{Competency Based Regenerative Medicine Curriculum.} Regenerative medicine is a branch of science that utilizes various tools, such as stem cells, biomaterials, and tissue engineering, to replace or regenerate damaged tissues and ultimately restore normal function to patients [22-24]. This field will have a huge impact on human health as it promises to cure diseases rather than treat the symptoms of a disease which is one of the reasons that regenerative medicine research is growing exponentially. The major topics in regenerative medicine include stem cells, biomaterials, cell therapies, tissue engineering, enabling technologies, and translational research, to name a few [24]. For the purposes of this review, to identify the most important techniques used in regenerative medicine, we compiled a diverse list of journals where articles in the regenerative medicine field are published (Table 1). The corresponding impact factor (IF) for each journal is provided, which is an important indicator of how much a journal is read. The impact factors of the journals reviewed here varied widely from 41.5 (Nature, second highest impact factor of all journals) to 2.5 and included a journal that had no impact factor due to its recent inception but was highly cited. Only techniques from articles in these journals related to regenerative medicine were included in this review.

\section{Results and Discussion}

2.1. Identification of Techniques Used in Regenerative Medicine Journal Articles Published in Top Journals in the Field. The regenerative medicine publications we reviewed from the journals listed in Table 1 produced 887 data points and identified 169 distinct techniques used in the articles reviewed (Supplemental Table 1 (see Supplementary Material available online at http://dx.doi.org/10.1155/2016/9343716)). Many of these techniques were only utilized in one of the studies we reviewed ((Figure 1) light green indicates one study). A few techniques were utilized in as many as 90 different studies, indicating these techniques were commonly used in 


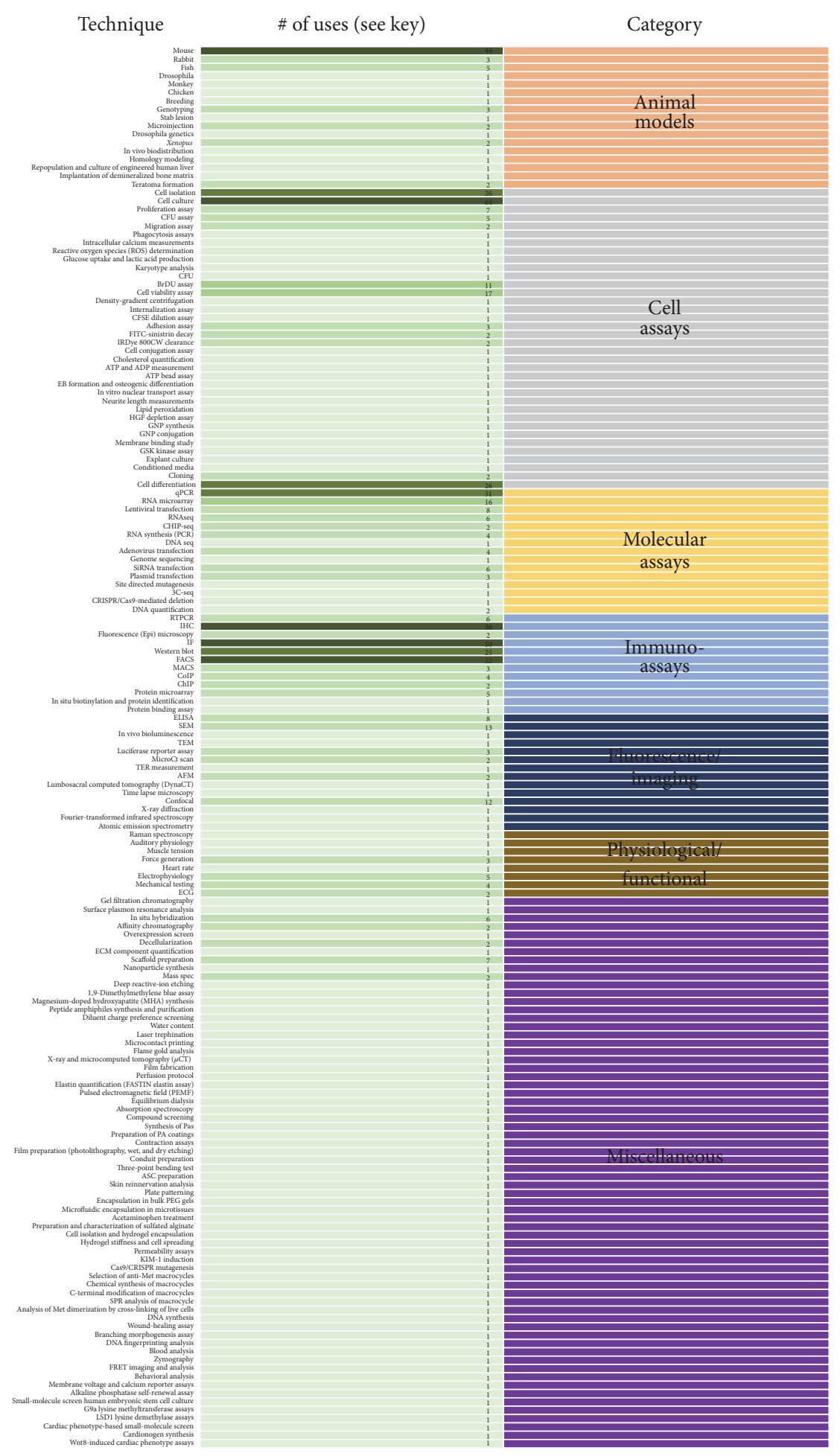

Key: number of journal articles that utilized this technique

$$
\begin{array}{ll}
31+ & 2 \text { to } 10 \\
21 \text { to } 30 & 1 \\
11 \text { to } 20 &
\end{array}
$$

FIGURE 1: Compiled list of techniques used in regenerative medicine publications. A total of 887 data points were collected from a review of over 150 regenerative medicine articles which led to the identification of 169 distinct techniques. Techniques appeared in as many as 90 articles and as few as 1 article. The identified techniques were organized into 7 categories including animal models, cell assays, molecular assays, immunoassays, fluorescence/imaging, and physiological/functional and miscellaneous. A comprehensive list of the techniques identified in this literature review is available in Supplemental Materials. 
TABLE 1: Journals that publish regenerative medicine research articles used in this review.

\begin{tabular}{|c|c|c|}
\hline Topic & Journal name & Impact factor \\
\hline \multirow{5}{*}{ Stem cells } & Cell Stem Cell & 23.6 \\
\hline & Cell Research & 12.4 \\
\hline & Developmental Cell & 9.7 \\
\hline & Stem Cell Translational Medicine & 5.7 \\
\hline & Stem Cell Reports & 5.4 \\
\hline \multirow{2}{*}{ Biomaterials } & Biomaterials & 8.6 \\
\hline & Acta Biomaterialia & 6.0 \\
\hline \multirow{2}{*}{ Tissue engineering } & Journal of Tissue Engineering and Regenerative Medicine & 5.2 \\
\hline & Tissue Engineering Part A & 4.6 \\
\hline \multirow{11}{*}{ General } & Nature & 41.5 \\
\hline & Science & 33.6 \\
\hline & Cell & 32.2 \\
\hline & Nature Communications & 11.5 \\
\hline & Molecular Therapy & 6.8 \\
\hline & Chemistry and Biology & 6.7 \\
\hline & Scientific Reports & 5.6 \\
\hline & Methods & 3.6 \\
\hline & Experimental and Molecular Medicine & 3.5 \\
\hline & International Journal of Oral Science & 2.5 \\
\hline & Molecular Therapy-Methods and Translational Development & * \\
\hline
\end{tabular}

${ }^{*}$ Newer journals that have not been assigned an impact factor.

the regenerative medicine field ((Figure 1) the darker green correlates with use in more studies). The most commonly used technique, observed in 90 different studies, was cell culture. The next most commonly used techniques include immunofluorescence followed closely by mouse studies, histochemistry, fluorescence activated cell sorting (FACS), and quantitative polymerase chain reaction (qPCR). This comprehensive list of techniques was organized into seven different categories, including animal studies, cell assays, molecular techniques, immunoassays, fluorescence/imaging techniques, physiological/functional assays, and miscellaneous techniques.

2.2. Most Commonly Utilized Techniques in Regenerative Medicine Articles Published in Top Journals in the Field. Of the techniques we identified in this review of regenerative medicine articles the top 20 included, cell culture, mouse studies, immunofluorescence, FACS, histochemistry, cell transfection, Western blot, quantitative polymerase chain reaction (qPCR), cell isolation, cell differentiation, ribonucleic acid (RNA) microarray, physiological studies, confocal microscopy, bromodeoxyuridine (BrDU) assay, scanning electron microscopy (SEM), proliferation assays, scaffold preparation, reverse transcriptase PCR (RTPCR), and RNA sequencing (Figure 2). Of the 887 technique data points identified in this literature review the top 20 accounted for 633 or greater than $70 \%$ of the data points, indicating that there is a small group of techniques used commonly by labs in the regenerative medicine field for publications.
2.3. Categorizing the Top Regenerative Medicine Techniques. The top 20 techniques used in regenerative medicine articles were categorized into four groups. These groups included immunoassays, molecular techniques, cell/animal models, and miscellaneous techniques which could not be classified into a specific group (Figure 3). Immunoassays comprised immunofluorescence, FACS, Western blot, and histochemistry which accounted for 217 of our technique data points. The skills required to gain competence in the immunoassays group include but are not limited to basic laboratory skills (pipetting, measuring, etc.), antibody, and chemical staining (immobilized and suspension). The molecular techniques category includes transfection, qPCR, RNA microarray, and RTPCR accounting for 123 of our technique data points. To be competent in this category individuals would also need basic laboratory skills along with PCR. The cell/animal model group included cell culture, mouse studies, cell isolation, and cell differentiation accounting for 246 data points. Competency in the cell/animal model group requires more advanced laboratory skills (cell culture, sterile technique, etc.) as well as the ability to work with research animals. Those techniques that did not fit into either of these categories included confocal and BrDU assays which accounted for 76 data points in this study. An understanding of laboratory practices, cell/tissue sample preparation, and microscopy would be essential to becoming proficient at these techniques.

2.4. Moving Forward. CBE will improve the value and accessibility of higher education for many students. In order to provide students with quality CBE programs we must (1) 


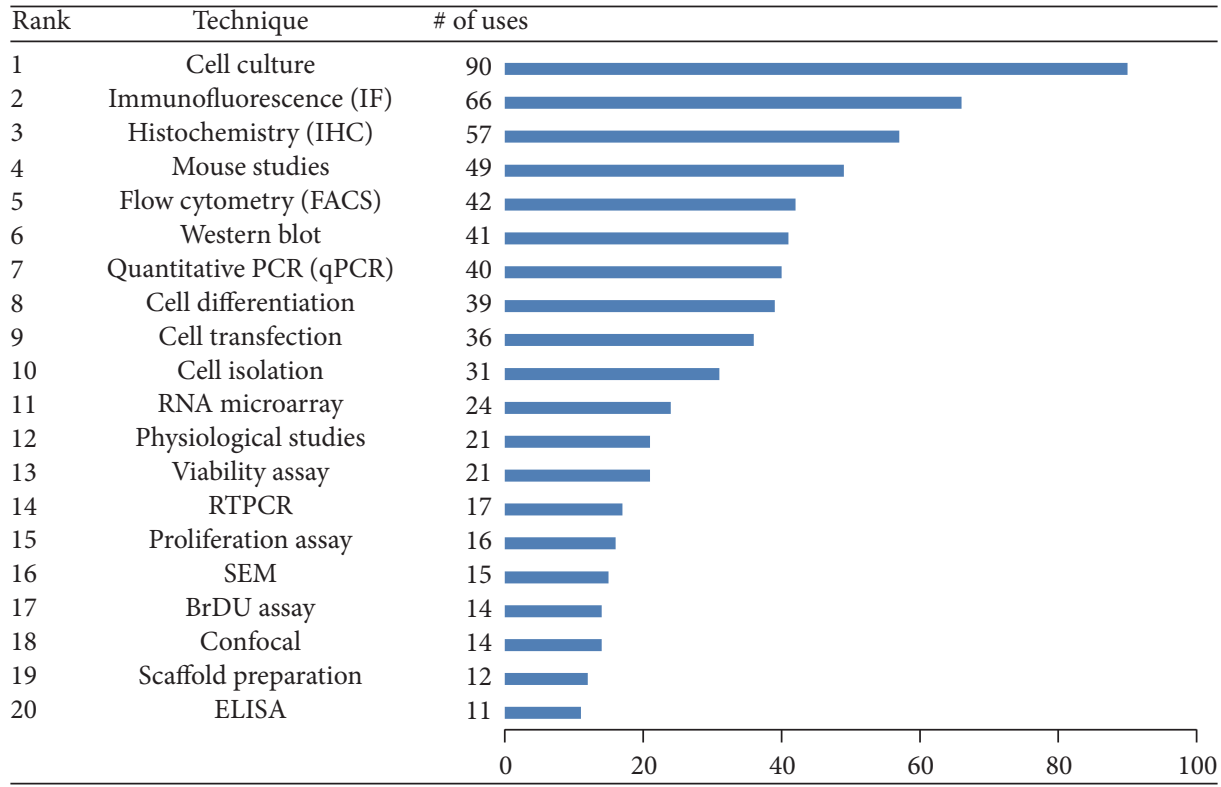

FIGURE 2: Top 20 techniques utilized in the reviewed regenerative medicine publications. The top 20 most common techniques identified through this literature review of regenerative medicine articles are listed in descending order. The most commonly used technique was cell culture which was used in 90 of the studies we investigated in this review. Of the data points collected the top 20 techniques comprised greater than $70 \%$ of the techniques used.

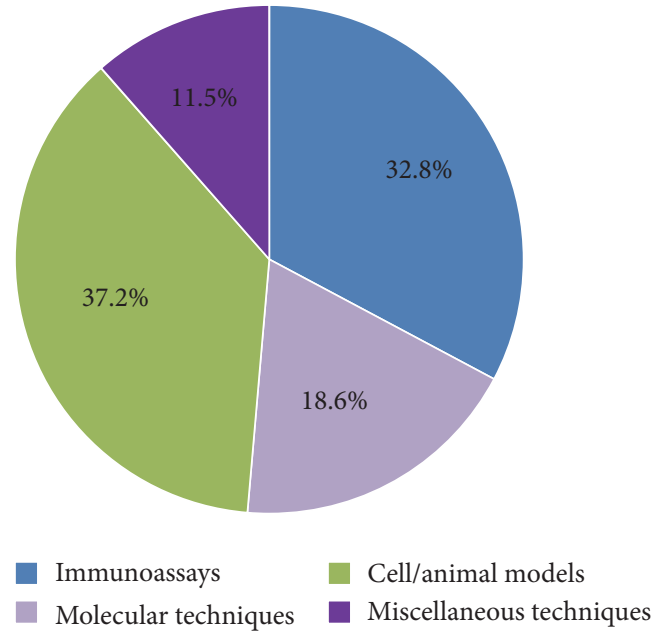

Figure 3: Categories represented by the top 15 regenerative medicine techniques. Immunoassays and cell/animal models were each used in 217 and 246 studies, respectively, compared to molecular and miscellaneous techniques which were used in 123 and 76 studies, respectively. The percentages of studies these categories represent are provided in the graph.

design programs that students can complete at their own pace, (2) develop innovative methods to deliver content to students, and perhaps most importantly (3) provide students with the most relevant and pertinent knowledge and skills associated with a given field. Additionally, CBE programs need to have clearly defined, concrete, and measurable objectives for each competency provided to students. Here we perform a literature review of the techniques used in studies published in top journals for the regenerative medicine field to provide proof of concept that this is an ideal method for identifying the most relevant competencies in a given field. By reviewing recent articles published in top journals in the field of regenerative medicine we were able to identify a list of techniques that are commonly used, thereby providing a roadmap for developing a competency based regenerative medicine curriculum. Identification of techniques through a review of recent literature guarantees that the techniques identified are currently used in regenerative medicine laboratories. Furthermore, the fact that articles are published in distinguished journals in the field ensures that these techniques are performed in successful and productive labs.

This review led to the identification of a large list of techniques utilized by regenerative medicine researchers. Of this list many techniques were only observed in one journal article, whereas the top 20 most commonly used techniques accounted for over $75 \%$ of the data points identified in this review. We interpret this as meaning a small set of techniques are utilized very frequently by researchers in the field of regenerative medicine. That being the case, we consider the most frequently used techniques to be among the most important ones for students to know and, therefore, the techniques that should be provided by a competency based regenerative medicine program. It would not benefit students to learn techniques that were only used in one of these studies since it would be unlikely that many employers are looking for those particular skills. Furthermore, even the labs that perform the uncommon techniques also performed many of the commonly used techniques in the large majority of articles reviewed here. This list of techniques will also 
help institutions prioritize the importance of specific competencies that should be delivered to students. Based on this data, we would emphasize the techniques identified in this review as being the most commonly utilized regenerative medicine techniques. This approach will have a better chance of preparing students with the skills that most employers currently need. In terms of how a competency based regenerative medicine curriculum could be developed based on this data, institutions would include the top 20 techniques in the required course materials and provide uncommon regenerative medicine techniques as optional electives.

The list of techniques provided here serves as an excellent guide for developing a competency based regenerative medicine program. However, it is extremely important for this list to be constantly updated through additional reviews, since the biomedical research field is rapidly advancing and new techniques are frequently developed. With the rapid progress being made in the fields of genomics and gene editing we do expect that these techniques will soon be on the top 20 list of techniques. There are numerous other techniques that are also advancing and becoming more common; therefore updating this list will help in developing programs that provide students with the most up to date and relevant training in their field. Using the literature review method we performed here will allow us to easily identify trending techniques.

There are some limitations associated with this technique including (1) potential selection bias depending on the journals utilized in this review, (2) certain journals that may publish more articles that utilize newer techniques thereby skewing our results, and (3) laboratories utilizing techniques based on availability of equipment or expertise rather than relevance to the field. Perhaps the best approach to identifying the most commonly utilized techniques in a field requires a combination of literature reviews and surveying experts in the field.

\section{Conclusion}

The educational landscape is constantly shifting and CBE systems are one-way educators that can adapt to the needs of students and provide efficient and effective programs to train the diverse populations of students entering higher education. Improving the delivery and optimizing the content of $\mathrm{CBE}$ are essential to staying ahead of the curve and using the literature review method identified here will be an excellent tool on this front.

\section{Competing Interests}

The authors declare no conflict of interests.

\section{References}

[1] M. Hart, "Competency-based education," Journal of the American Dietetic Association, vol. 69, no. 6, pp. 616-620, 1976.

[2] F. B. Wiebusch, J. K. Wittermann, and S. A. Saroff, "Report of a competency based education model in continuing education," Virginia Dental Journal, vol. 53, no. 3, pp. 15-26, 1976.
[3] J. R. Frank, R. Mungroo, Y. Ahmad, M. Wang, S. De Rossi, and T. Horsley, "Toward a definition of competency-based education in medicine: a systematic review of published definitions," Medical Teacher, vol. 32, no. 8, pp. 631-637, 2010.

[4] P. Harris, L. Snell, M. Talbot, and R. M. Harden, "Competencybased medical education: implications for undergraduate programs," Medical Teacher, vol. 32, no. 8, pp. 646-650, 2010.

[5] R. M. Pigg Jr., "A national study of competency based health education programs," Health Education, vol. 7, no. 4, pp. 15-16, 1976.

[6] G. K. Thurman and M. K. Sanders, "Competency-based education versus traditional education: a comparison of effectiveness," Radiologic Technology, vol. 59, no. 2, pp. 164-169, 1987.

[7] E. J. Harvey and V. C. McAlister, "Cross-specialty training in the era of competency-based education," Canadian Journal of Surgery, vol. 58, no. 6, pp. 364-366, 2015.

[8] C. P. van der Vleuten, "Competency-based education is beneficial for professional development," Perspectives on Medical Education, vol. 4, no. 6, pp. 323-325, 2015.

[9] K. Walsh, "Competency-based medical education: the next steps," Indian Pediatrics, vol. 52, no. 10, p. 905, 2015.

[10] N. Goodyear, S. Kim, M. Reeves, and M. L. Astion, "A 2year study of Gram stain competency assessment in 40 clinical laboratories," American Journal of Clinical Pathology, vol. 125, no. 1, pp. 28-33, 2006.

[11] R. E. Hawkins, C. M. Welcher, E. S. Holmboe et al., "Implementation of competency-based medical education: are we addressing the concerns and challenges?" Medical Education, vol. 49, no. 11, pp. 1086-1102, 2015.

[12] P. Lebensohn, B. Kligler, S. Dodds et al., "Integrative medicine in residency education: developing competency through online curriculum training," Journal of Graduate Medical Education, vol. 4, no. 1, pp. 76-82, 2012.

[13] D. S. Watson, "Developing a competency-based education program for nurse-monitored sedation," Seminars in Perioperative Nursing, vol. 1, no. 4, pp. 224-231, 1992.

[14] L. A. McEwen, D. Harris, R. F. Schmid, J. Vogel, T. Western, and P. Harrisoncorresponding, "Evaluation of the redesign of an undergraduate cell biology course," CBE-Life Sciences Education, vol. 8, no. 1, pp. 72-78, 2009.

[15] K. R. Zinn, C. J. Anderson, M. Bradbury et al., "Components of a curriculum for molecular imaging scientists," Journal of Nuclear Medicine, vol. 52, no. 4, pp. 650-656, 2011.

[16] M. J. Lipp, "A process for developing assessments and instruction in competency-based dental education," Journal of Dental Education, vol. 74, no. 5, pp. 499-509, 2010.

[17] S. M. Johnstone and L. Soares, "Principles for developing competency-based education programs," Change: The Magazine of Higher Learning, vol. 46, no. 2, pp. 12-19, 2014.

[18] L. P. D. Jackie Krause and C. Schedler, "Competency-based education: a framework for measuring quality courses," Online Journal of Distance Learning Administration, vol. 18, no. 1, 2015.

[19] B. F. Walker, N. J. Stomski, J. J. Hebert, and S. D. French, "Evidence-based practice in chiropractic practice: a survey of chiropractors' knowledge, skills, use of research literature and barriers to the use of research evidence," Complementary Therapies in Medicine, vol. 22, no. 2, pp. 286-295, 2014.

[20] C. Fekete, W. Segerer, A. Gemperli, and M. W. Brinkhof, "Participation rates, response bias and response behaviours in the community survey of the Swiss Spinal Cord Injury Cohort Study (SwiSCI)," BMC Medical Research Methodology, vol. 15, article 80, 2015. 
[21] A. Scott, S.-H. Jeon, C. M. Joyce et al., "A randomised trial and economic evaluation of the effect of response mode on response rate, response bias, and item non-response in a survey of doctors," BMC Medical Research Methodology, vol. 11, article 126, 2011.

[22] Q. A. Rafiq, I. Ortega, S. I. Jenkins et al., "The early career researcher's toolkit: translating tissue engineering, regenerative medicine and cell therapy products," Regenerative Medicine, vol. 10, no. 8, pp. 989-1003, 2015.

[23] S. L. Rego, E. Zakhem, G. Orlando, and K. N. Bitar, "Bioengineered human pyloric sphincters using autologous smooth muscle and neural progenitor cells," Tissue Engineering Part A, vol. 22, no. 1-2, pp. 151-160, 2016.

[24] A. Atala and S. Murphy, "Regenerative medicine," The Journal of the American Medical Association, vol. 313, no. 14, pp. 1413-1414, 2015. 


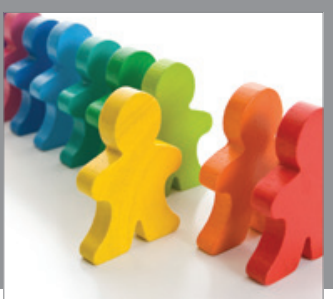

Autism

Research and Treatment
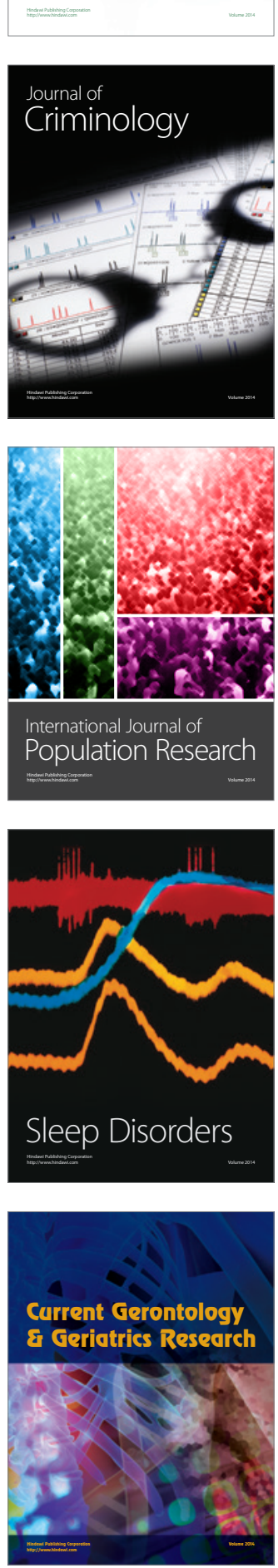

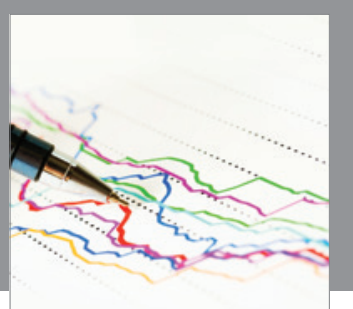

Economics

Research International
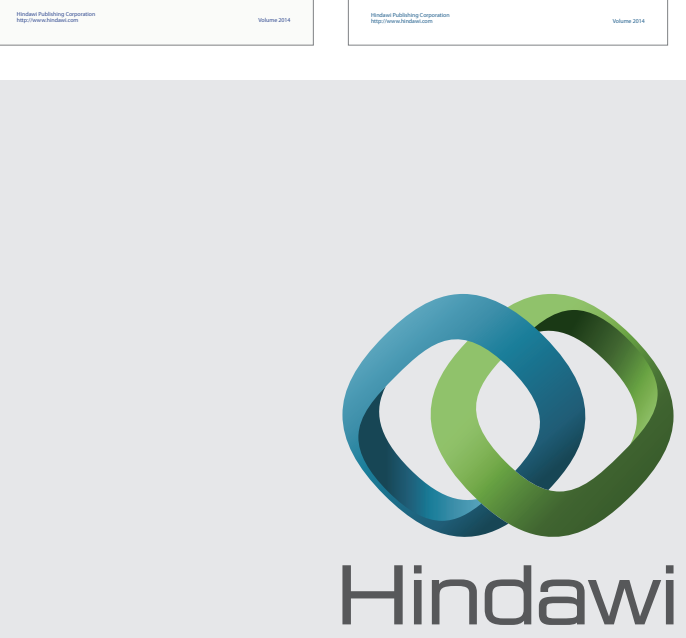

Submit your manuscripts at

http://www.hindawi.com
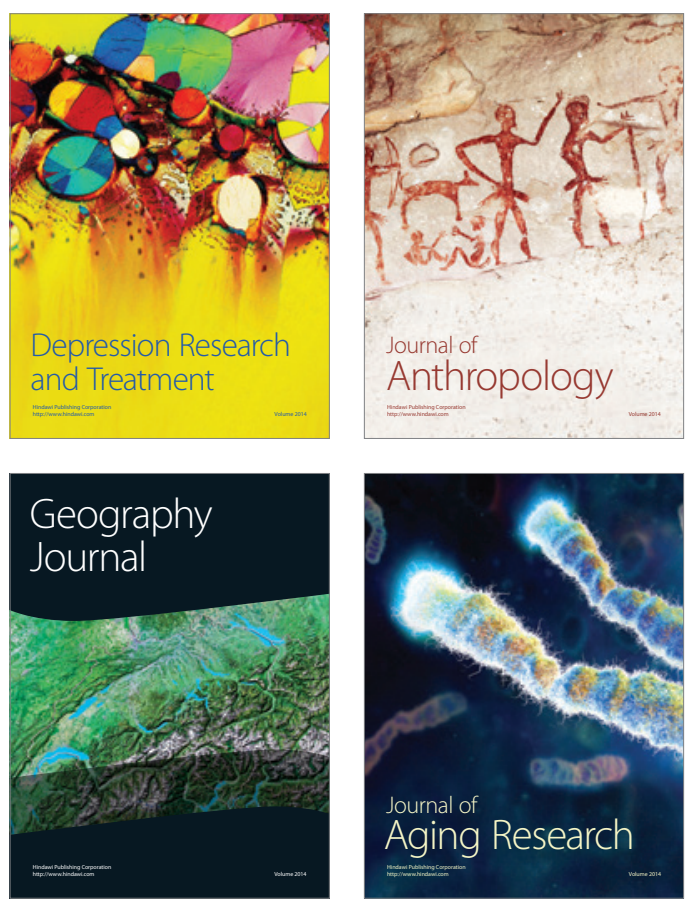
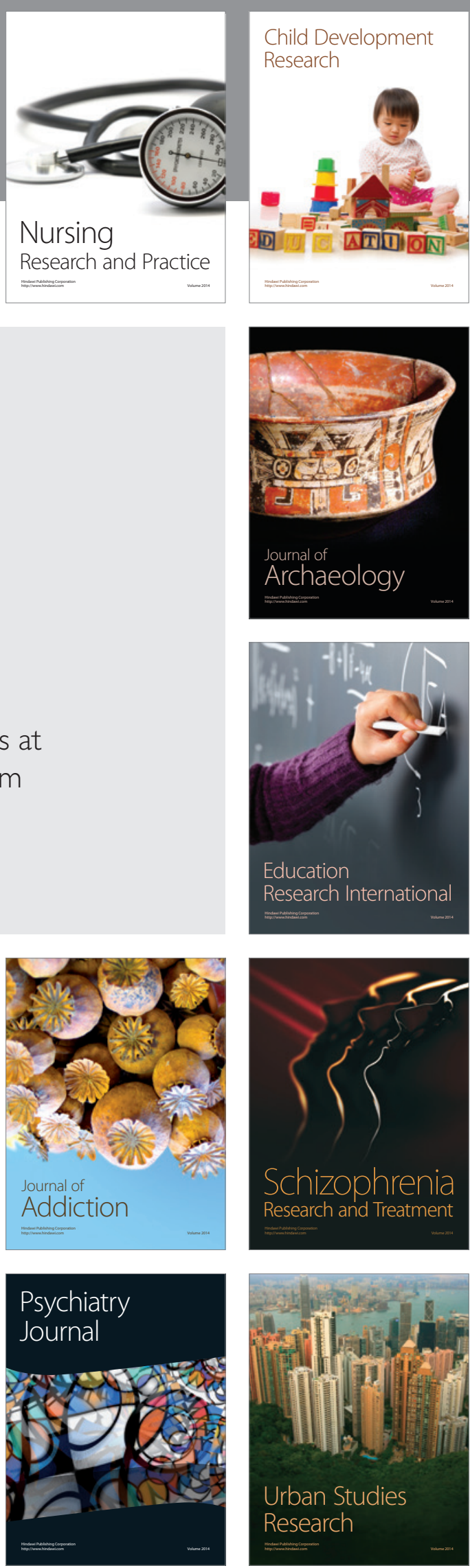